\title{
High-Throughput Screening Assay for the Identification of Compounds Enhancing Collagenous Extracellular Matrix Production by ATDC5 Cells
}

Citation for published version (APA):

Le, B. Q., Fernandes, H., Bouten, C. V. C., Karperien, M., van Blitterswijk, C., \& de Boer, J. (2015). HighThroughput Screening Assay for the Identification of Compounds Enhancing Collagenous Extracellular Matrix Production by ATDC5 Cells. Tissue Engineering. Part C. Methods , 21(7), 726-736. https://doi.org/10.1089/ten.tec.2014.0088

Document status and date:

Published: 01/07/2015

DOI:

10.1089/ten.tec.2014.0088

Document Version:

Publisher's PDF, also known as Version of record

Document license:

Taverne

Please check the document version of this publication:

- A submitted manuscript is the version of the article upon submission and before peer-review. There can be important differences between the submitted version and the official published version of record.

People interested in the research are advised to contact the author for the final version of the publication, or visit the DOI to the publisher's website.

- The final author version and the galley proof are versions of the publication after peer review.

- The final published version features the final layout of the paper including the volume, issue and page numbers.

Link to publication

\footnotetext{
General rights rights.

- You may freely distribute the URL identifying the publication in the public portal. please follow below link for the End User Agreement:

www.umlib.nl/taverne-license

Take down policy

If you believe that this document breaches copyright please contact us at:

repository@maastrichtuniversity.nl

providing details and we will investigate your claim.
}

Copyright and moral rights for the publications made accessible in the public portal are retained by the authors and/or other copyright owners and it is a condition of accessing publications that users recognise and abide by the legal requirements associated with these

- Users may download and print one copy of any publication from the public portal for the purpose of private study or research.

- You may not further distribute the material or use it for any profit-making activity or commercial gain

If the publication is distributed under the terms of Article 25fa of the Dutch Copyright Act, indicated by the "Taverne" license above, 


\title{
High-Throughput Screening Assay for the Identification of Compounds Enhancing Collagenous Extracellular Matrix Production by ATDC5 Cells
}

\author{
Bach q. Le, BSc, Hugo Fernandes, PhD, ${ }^{1}$ Carlijn V.C. Bouten, PhD, ${ }^{2}$ Marcel Karperien, PhD, ${ }^{3}$ \\ Clemens van Blitterswijk, $\mathrm{PhD}^{1}$, and Jan de Boer, $\mathrm{PhD}^{1}$
}

\begin{abstract}
Extracellular matrix (ECM) provides not only structural support to the cells but also signals that regulate their fate. In many tissue engineering approaches, it remains challenging to achieve the right amount and type of ECM secreted by the cells to faithfully mimic the native tissue. In this article, we describe how to design and perform a high-throughput assay to screen for molecules capable of enhancing collagenous ECM (cECM) production. We chose ATDC5 cells to validate the assay since we want to use this chondrogenic cell line later for tissue engineering of hypertrophic cartilage. We used a fluorescently labeled collagen-binding probe to quantify total collagen content in ATDC5 cultures. The LOPAC ${ }^{1280}$ library of pharmaceutically active compounds was screened using insulin (a known inducer of cECM in ATDC5 cells) as positive control. After screening and validation, the small-molecule tetradecylthioacetic acid (TTA) was shown to enhance cECM production by ATDC5 cells at both gene expression and protein level. Moreover, when combined with insulin, TTA showed a synergistic effect on cECM production. In contrast, exposure of human primary chondrocytes and human mesenchymal stromal cells to TTA did not induce cECM secretion. In conclusion, we have developed an HTS assay to screen for compounds capable of enhancing cECM production and discovered TTA as a potent enhancer of cECM secretion by ATDC5 cells.
\end{abstract}

\section{Introduction}

$\mathbf{M}$ ANY DIFFERENT CELL types secrete an extracellular matrix (ECM), which provides mechanical support and serves as a signaling hub conveying signals from the cells to the surrounding environment and vice versa. Some of these signals control important biological functions such as cell viability and cell fate. ${ }^{1,2}$ In tissue engineering, a common procedure is to isolate cells from their native tissue while leaving behind the ECM. The cells are then expanded to increase numbers, and are reaggregated to mimic a three-dimensional (3D) environment, either by the use of a scaffold or simply by cell aggregation. Ideally, the cells in the tissue-engineered construct will adhere, proliferate, and secrete ECM. The nature of secreted ECM can be, to a certain extent, controlled by the signals provided by the scaffold (e.g., topographical cues) or by soluble signals to which the cells are exposed (e.g., growth factors or small molecules). ${ }^{3-5}$
In bone tissue engineering through endochondral ossification, the amount of hypertrophic cartilage matrix in the tissueengineered construct is crucial for successful bone formation. We reported previously a method to efficiently generate bone in vivo using mouse embryonic stem cells predifferentiated into hypertrophic chondrocytes in vitro. ${ }^{6}$ The amount of bone detected in the construct after implantation was highly correlated with the amount of hypertrophic cartilage observed in vitro. Interestingly, the capacity of hypertrophic cartilage matrix to induce bone formation in vivo is not only limited to living cartilage. In 1958, Bridge and Pritchard performed a series of experiments, in which devitalized tissues were placed under the kidney capsules or implanted subcutaneously in the ears of rabbits. Tissues containing hypertrophic cartilage, which were devitalized with alcohol, acetone, and $\mathrm{HCl}$, or heated to $55^{\circ} \mathrm{C}$ consistently, formed bone. ${ }^{7}$ Later, Marshall Urist also observed new bone formation when a boiled fracture callus was transplanted into the anterior chamber of a rat's eye. ${ }^{8-10}$ This interesting phenomenon can

\footnotetext{
${ }^{1}$ Department of Tissue Regeneration, MIRA Institute for Biomedical Technology and Technical Medicine, University of Twente, Enschede, The Netherlands.

${ }^{2}$ Laboratory for Cell and Tissue Engineering, Department of Biomedical Engineering, Eindhoven University of Technology, Eindhoven, The Netherlands.

${ }^{3}$ Department of Developmental BioEngineering, MIRA Institute for Biomedical Technology and Technical Medicine, University of Twente, Enschede, The Netherlands.
} 
be exploited to produce off-the-shelf bone-inducing hypertrophic cartilage since devitalized allografts provoke a much milder immune response compared with viable tissue. ${ }^{11,12}$ We attempt to repeat the devitalized cartilage experiment, but instead of using natural hypertrophic tissue, such as growth plate cartilage or fracture callus, we try to tissue engineer hypertrophic cartilage in vitro to better control the state of hypertrophy. However, one final hurdle remains: production of sufficient amounts of ECM in vitro.

Previously, we used high-throughput screening (HTS) to search for small molecules that can modulate the cellular response of certain cell types. For instance, using HTS, we identified several compounds that improve osteogenic differentiation of the human mesenchymal stromal cell $(\mathrm{hMSC})^{13}$ and discovered that the small-molecule phenanthroline is very efficient in inducing angiogenesis. ${ }^{14}$

In this article, we designed and performed an HTS assay to search for compounds that can enhance ECM production. We chose the chondrogenic cell line, ATDC5, for this HTS assay because it is a well-established cell line able to produce collagenous ECM (cECM) while growing in twodimensional (2D) culture on normal tissue culture-treated polystyrene surface, which simplifies the detection method. More importantly, ATDC5 cells can consistently differentiate into hypertrophic chondrocytes and produce hypertrophic cartilage matrix in vitro, ${ }^{15}$ which can be used later for our devitalized cartilage strategy. To quantify the amount of collagenous matrix produced by the cells, we use a fluorescently labeled collagen-binding probe, which has affinity for both fibrillar and nonfibrillar collagen. ${ }^{16,17}$ This property, that is, not specific to any particular type of collagen, was exploited to measure the total amount of $\mathrm{CECM}$ produced by the cells. Using this assay, we identified and validated tetradecylthioacetic acid (TTA) as a compound capable of enhancing $\mathrm{CECM}$ production by ATDC5 cells.

\section{Materials and Methods}

\section{Cell culture}

The ATDC5 cell line was obtained from the RIKEN cell bank (Japan) and maintained in basic medium (BM), which consists of a 1:1 mixture of Dulbecco's modified Eagle's medium (DMEM) and Ham's F-12 supplied with GlutaMAX $^{\text {TM }}$ (DMEM/F-12 GlutaMAX), 5\% v/v fetal bovine serum (FBS; Lonza), 0.2 mM L-ascorbic acid-2 phosphate (ASAP), $100 \mathrm{U} / \mathrm{mL}$ of penicillin, and $100 \mu \mathrm{g} / \mathrm{mL}$ of streptomycin (PS). Cells were grown at $37^{\circ} \mathrm{C}$ in a humid atmosphere with $5 \% \mathrm{CO}_{2}$. The medium was refreshed twice a week and cells were used for further subculturing or cryopreservation upon reaching near confluence. To stimulate chondrogenesis and ECM production, BM was replaced with insulin medium (IM) consisting of BM supplemented with $1 \times$ insulin, transferrin, selenium solution (ITS) to yield a final concentration of $10 \mu \mathrm{g} / \mathrm{mL}$ insulin, $5.5 \mu \mathrm{g} / \mathrm{mL}$ transferrin, and $3.87 \times 10^{-8} \mathrm{M}$ sodium selenite. ${ }^{18}$

Primary human mesenchymal stem cells (hMSCs, three donors) and human primary articular chondrocytes (hPCs, three donors) were obtained and isolated as described previously. ${ }^{19,20}$ The use of bone marrow aspirates and human knee biopsies was approved by the Medical Ethics Committee of Medisch Spectrum Twente, and informed consent was obtained from all patients. hMSCs were expanded at the initial seeding density of 1000 cells $/ \mathrm{cm}^{2}$ in proliferation medium consisting of $\alpha$-MEM, $10 \%$ v/v FBS (Lonza), $2 \mathrm{mM}$ L-glutamine, $0.2 \mathrm{mM}$ ASAP, PS, and $1 \mathrm{ng} / \mathrm{mL}$ recombinant human basic fibroblast growth factor (AbD Serotec). For chondrogenic differentiation, hMSCs (passage 2) were seeded at $2.5 \times 10^{5}$ cells per well in Cellstar U-shaped 96-well plates (Greiner Bio-one) and centrifuged for $3 \mathrm{~min}$ at $300 \mathrm{~g}$ to form pellets. Chondrogenic medium for hMSCs consists of DMEM high glucose, $100 \mu \mathrm{g} / \mathrm{mL}$ sodium pyruvate, $0.2 \mathrm{mM}$ ASAP, PS, $1 \times$ ITS, $0.1 \mu \mathrm{M}$ dexamethasone (Dex), and $10 \mathrm{ng} / \mathrm{mL}$ transforming growth factor 3 (TGF $\beta 3$; R\&D systems, 243-B3010). hMSC pellets were cultured for 28 days, and the medium was changed two to three times per week. hPCs were expanded in chondrocyte proliferation medium consisting of DMEM, $10 \%$ v/v FBS, $1 \times$ nonessential amino acid, $0.2 \mathrm{mM}$ ASAP, $0.4 \mathrm{mM}$ proline, and PS. For chondrogenic differentiation, hPCs (passage 1) were seeded at 10,000 cells $/ \mathrm{cm}^{2}$ in six-well plates. Chondrogenic medium for hPCs was the same as for hMSCs with the addition of $2 \mathrm{mM} \mathrm{L}$-glutamine. hPCs were cultured for 14 days, and the medium was changed two to three times per week. All media and medium supplements were purchased from Life Technologies; all common chemicals were purchased from Sigma-Aldrich, unless otherwise stated.

\section{Collagen probe CN35-AF488 assay}

Cells were cultured on a 96-well black/clear imaging plate (BD Bioscience; 353219). Per time point, the culture medium was replaced with staining medium, which consists of $0.25 \mathrm{mM}$ CN35-AF488 diluted in DMEM/12 without supplements. CN35-AF488 consists of a collagen-binding protein domain (CNA35) conjugated to the fluorescent dye, Alexa Fluor 488.16,17 Using solid-phase binding assays and immunohistological staining, Krahn et al. showed that the collagen probe bound relatively well to all types of collagen that were tested with a different affinity for the different collagen types (I-VI), but did not bind to any of the other ECM proteins..$^{16}$ The plates were incubated at $37^{\circ} \mathrm{C}$ in a humid atmosphere with $5 \% \mathrm{CO}_{2}$ on an orbital shaker $(20 \mathrm{rpm})$ for $1 \mathrm{~h}$. After that, the plates were washed thrice with PBS (Gibco) to remove the unbound probe. Then, total fluorescent intensity of CNA35-AF488 was measured at $520 \mathrm{~nm}$ on a Victor plate reader (PerkinElmer).

\section{High-throughput screen}

ATDC5 cells were seeded at 5000 cells $/ \mathrm{cm}^{2}$ in a 96-well black/clear imaging plate (BD Bioscience; 353219) in BM and allowed to proliferate for 3 days. Then, the 1280 test compounds (LOPAC1280; Sigma Aldrich) were added at a final concentration of $4.5 \mu \mathrm{M}$ in $\mathrm{BM}$. BM and IM were used as negative (NC) and positive (PC) control, respectively. DMSO, the solvent used in the library at $0.25 \% \mathrm{v} / \mathrm{v}$, was added to the controls. The medium, including compounds, was changed after 3 days, and after 6 days, the collagen probe CNA35AF488 assay was performed. A compound was defined as a hit if its signal was greater than the mean plus three times standard deviation of the negative controls in the same plate.

\section{Hits validation}

Three different concentrations of each hit compound were tested for their capacity to enhance ECM production. The 
experimental protocol was the same as the primary screen, with the exception that each compound and concentration was tested in triplicate. A PrestoBlue assay was performed to assess the effect of the compound on metabolic activity, representing cell viability. Briefly, BM containing $10 \%(\mathrm{v} / \mathrm{v})$ PrestoBlue solution (Invitrogen) was added and incubated at $37^{\circ} \mathrm{C}$ for $1 \mathrm{~h}$. Then, fluorescence was measured at $590 \mathrm{~nm}$ on a Victor plate reader (PerkinElmer). The concentration that induced the highest secretion of cECM without affecting the metabolic activity was selected for subsequent experiments.

\section{Gene expression analysis}

ATDC5 cells $\left(5000\right.$ cells $\left./ \mathrm{cm}^{2}\right)$ and hPCs $(10,000$ cells/ $\mathrm{cm}^{2}$ ) were seeded in triplicate in six-well plates in BM and allowed to attach overnight. hMSCs $\left(2.5 \times 10^{5}\right.$ cells per well $)$ were aggregated in U-shaped 96-well plates in hMSC chondrogenic differentiation medium and allowed to form pellets overnight. Then, the compounds were added, and the medium was changed two to three times per week. At selected time points, RNA was isolated using an RNA II nucleospin RNA isolation kit (Machery Nagel). For chondrogenic differentiation of hMSCs, three pellets were pooled for RNA isolation. RNA concentrations were measured using an ND100 spectrophotometer (Nanodrop1000). cDNA was synthesized from $1 \mu \mathrm{g}$ of RNA using iScript (BioRad) according to the manufacturer's protocol. qPCR was performed using $50 \mathrm{ng}$ of cDNA, $0.4 \mu \mathrm{M}$ of each forward and reverse primer (Sigma Genosys), and 1x SensiMix SYBR and Fluorescein master mix (Bioline). Primer sequences are shown in Supplementary Table S2 (Supplementary Data are available online at www.liebertpub.com/tec). Real-time qPCR was performed in a Biorad My IQ5 machine (Biorad). Data were analyzed using the fit point method of My IQ5 software. The baseline was calculated automatically by the software at the lower loglinear part above baseline noise, and the crossing temperature (Ct value) was determined. $\mathrm{Ct}$ values were normalized to the Beta-2 microglobulin (B2M) housekeeping gene, and $\Delta \mathrm{Ct}$ (Ct, control-Ct, sample) was used to calculate the upregulation in gene expression.

\section{Total glycosaminoglycan quantification assay}

Glycosaminoglycan (GAG) assay was performed as described previously. ${ }^{20}$ Briefly, samples were washed with PBS and frozen at $-80^{\circ} \mathrm{C}$. Subsequently, they were digested with $1 \mathrm{mg} / \mathrm{mL}$ proteinase K (Sigma-Aldrich) in Tris/EDTA buffer ( $\mathrm{pH} 7.6$ ) containing $18.5 \mathrm{mg} / \mathrm{mL}$ iodoacetamide and $1 \mathrm{mg} / \mathrm{mL}$ pepstatin A (Sigma-Aldrich) overnight at $56^{\circ} \mathrm{C}$. GAG content was spectrophotometrically determined with 9-dimethylmethylene blue chloride (DMMB; Sigma-Aldrich) staining in PBE buffer $\left(14.2 \mathrm{~g} / \mathrm{L} \mathrm{Na} \mathrm{Na}_{2} \mathrm{HPO} 4\right.$ and $3.72 \mathrm{~g} / \mathrm{L}$ $\mathrm{Na}_{2}$ EDTA at $\mathrm{pH}$ 6.5) with a microplate reader (Bio-TEK instruments) at $520 \mathrm{~nm}$. The standard curve for the GAG analysis was generated using chondroitin sulfate A (from bovine trachea; Sigma-Aldrich).

\section{Hydroxyproline assay}

A hydroxyproline assay kit was purchased from Biovision (K555-100). The assay was performed according to the manufacturer's protocol. Briefly, samples (cell layer) in triplicate were retrieved from tissue culture flasks at weeks
$1,2,3$, and 4 using cell scrapers and transferred to pressuretight Teflon-capped vials for hydrolysis in $6 \mathrm{M} \mathrm{HCl}$ for $3 \mathrm{~h}$ at $120^{\circ} \mathrm{C}$. Lysates were transferred to a 96-well plate and dried under vacuum. Chloramine $\mathrm{T}$ and 4-(Dimethylamino)benzaldehyde (DMAB) reagent were added to each sample and incubated for $90 \mathrm{~min}$ at $60^{\circ} \mathrm{C}$. Absorbance at $560 \mathrm{~nm}$ was measured in a microplate reader (Bio-TEK instruments). The standard curve was generated using 4-hydroxyproline as indicated in the manufacturer's protocol.

\section{Statistics}

One-way or two-way ANOVA with Bonferroni's posttest and Pearson correlation analysis were performed using GraphPad Prism version 6.02 for Windows, GraphPad Software, www.graphpad.com.

\section{Results}

\section{ECM production in differentiating ATDC5 cells}

ATDC5 cells produce high amounts of cECM in 2D culture. To characterize our ATDC5 cell line, we cultured the cells for 3-4 weeks in BM and IM and analyzed their morphology and the expression profile of cECM-related genes. Figure 1A shows the morphological changes of the ATDC5 cell layer upon exposure to IM over 3 weeks. At day 7 , the cell layer morphology was similar for both conditions. At day 14, the ATDC5 cell layer treated with insulin developed many aggregates, which stain positive for Alcian Blue (data not shown), and further developed into nodules after 21 days. In contrast, in BM, small aggregates appeared at day 21, 1 week later than in IM. However, when cultured for up to 40 days, these aggregates did not progress into nodules as seen in the IM (data not shown). The ATDC5 nodules did not stain positive for Alizarin Red (data not shown) unless the medium was switched to mineralization medium supplemented with calcium and phosphate salts. Gene expression analysis showed an increase in mRNA levels for collagen type II and type X in BM and IM over a 4-week time course (Fig. 1B). After 1 week, IM led to a 5 -fold increase in collagen type II expression compared with $\mathrm{BM}$, which increased to a 13-fold change upon 2 weeks and decreased thereafter to its basal level. A similar profile was observed for collagen type $\mathrm{X}$ expression (although in this case the fold change between the conditions reached a maximum of 60 at 2 weeks). A delay in the expression of both genes was observed in BM with maximum expression observed after 4 weeks instead of two for IM.

\section{Quantification of total collagen}

To quantify the total collagen content, we stained the cells with the CNA35-AF488 collagen probe. Figure 2A shows fluorescent images of ATDC5 cells, grown in BM and IM for 10 days, stained with the collagen probe. While ATDC5 cells also produce a basal level of collagen in the BM, the collagen network becomes much denser in the IM. The time point day 10 was chosen in our preliminary experiments; after this time, the cell layer in IM was already too thick and could detach easily in the staining and washing steps, affecting the fluorescent quantification. To demonstrate the capacity of the collagen probe assay, we compared it with a common GAG assay in a dose-response experiment of 


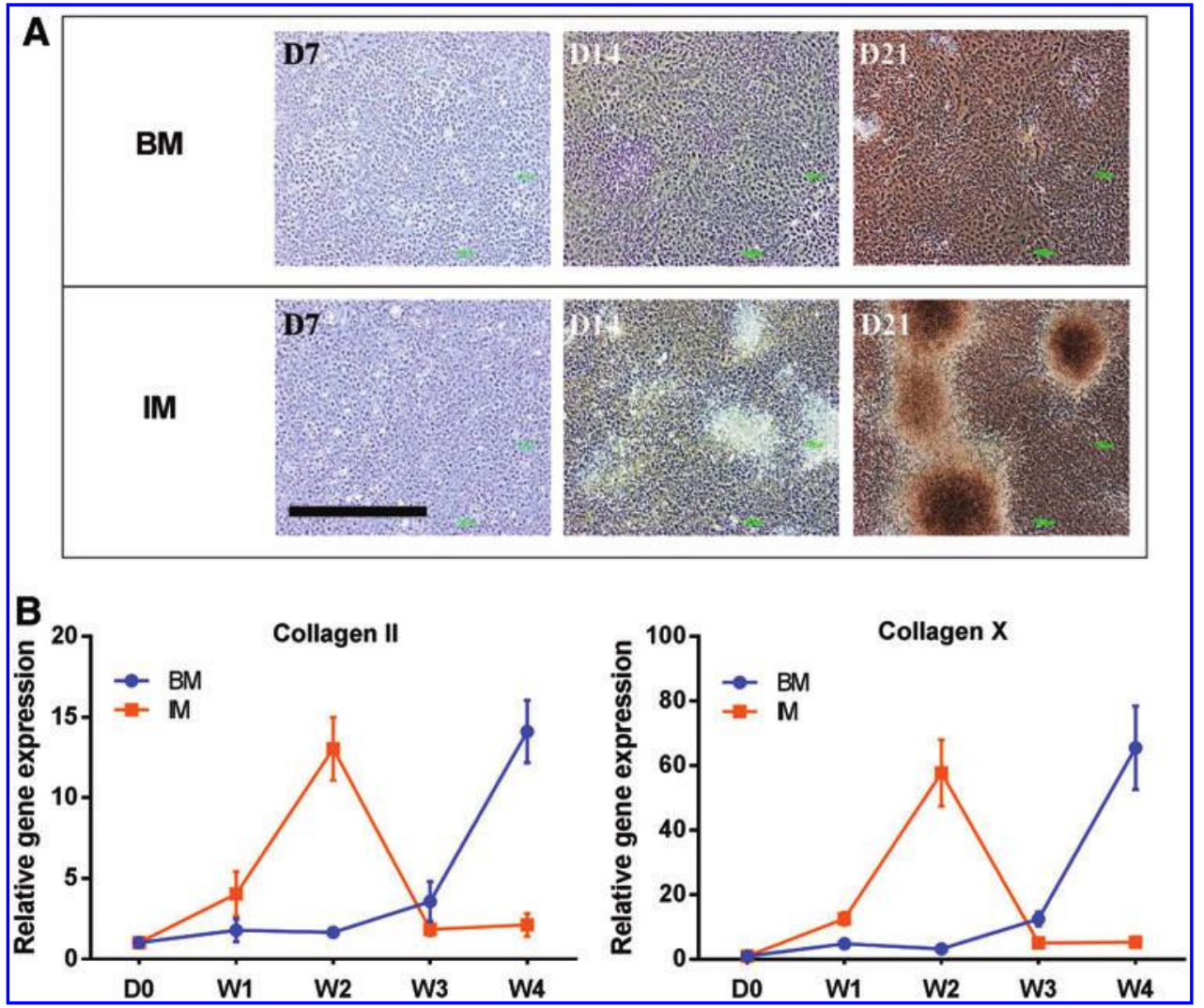

FIG. 1. Characterization of ATDC5 cell line. (A) Representative microscopy pictures of ATDC5 cultured on treated polystyrene surface (TCP) at days (D) 7, 14, and 21 in basic medium (BM) (top panel) or insulin medium (IM) (bottom panel). Scale bar represents $1 \mathrm{~mm}$. (B) Gene expression profile of collagen type II and X in ATDC5 5 cultured on TCP in $\mathrm{BM}$ (blue line) or IM (red line) at $\mathrm{D} 0$, weeks (W) 1,2 , 3 , and 4. $(n=3)$. Color images available online at www.liebertpub.com/tec

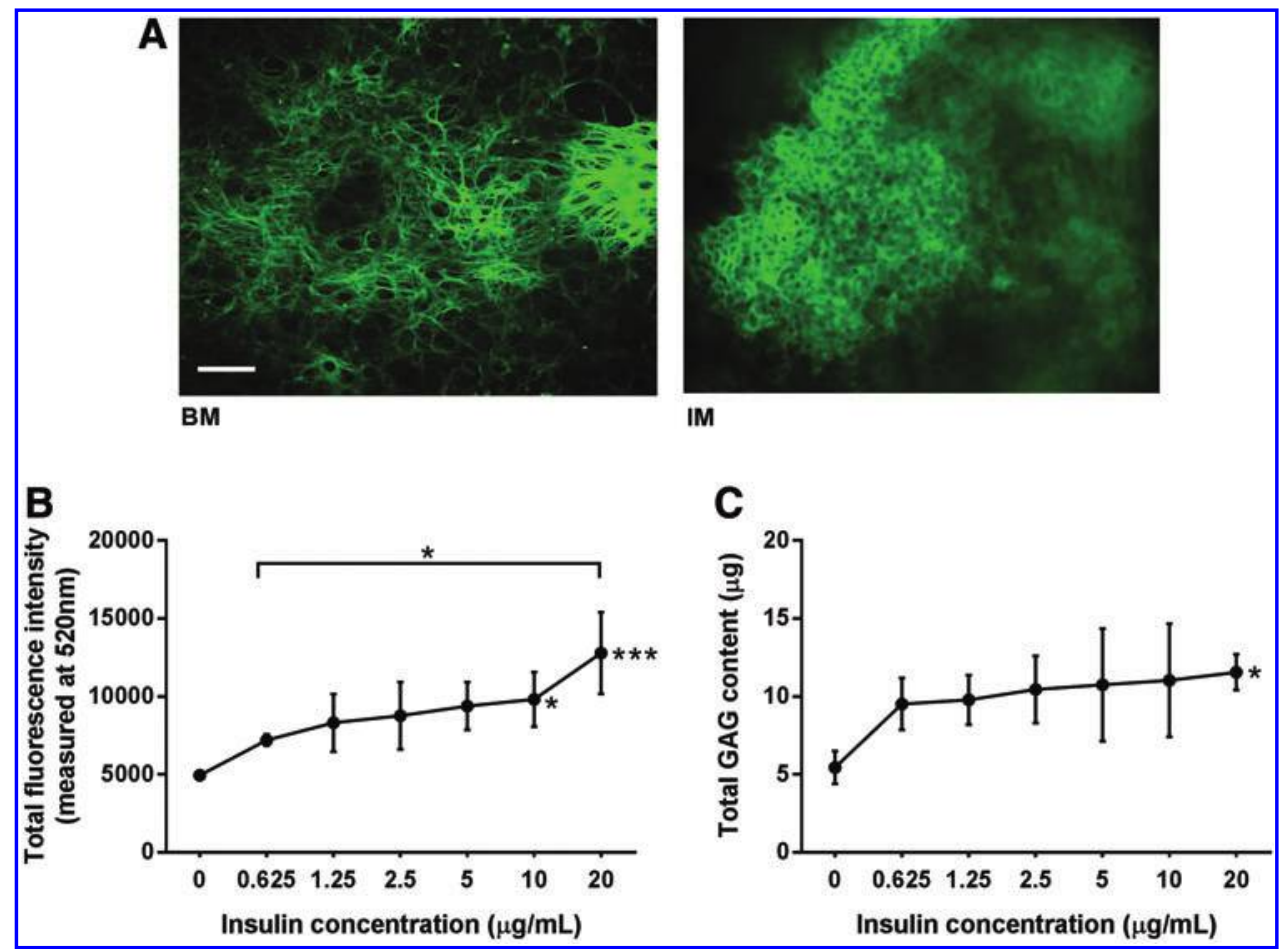

FIG. 2. Sensitivity of collagen probe CNA35-Alexa488 to detect extracellular matrix (ECM). (A) Fluorescence images of ATDC5 cultured on TCP for 10 days in BM or IM, stained with collagen probe CNA35-Alexa488, and visualized using a BD pathway 432 microscope. Scale bar represents $100 \mu \mathrm{m}$. Dose response of ATDC5 to insulin after 10 days cultured on TCP, (B) stained with collagen probe CNA35-Alexa488, and total fluorescence intensity measured by a Victor plate reader, or $(\mathbf{C})$ quantified by GAG assay. Error bars represent standard deviation $(n=3)$. Statistical analysis was performed using one-way ANOVA and Bonferroni's post-test. $(*)$ denotes $p<0.05$ compared with basic $(0 \mu \mathrm{g} / \mathrm{mL}$ insulin) and (***) denotes $p<0.001$ compared with basic. Color images available online at www.liebertpub.com/tec 
insulin on ATDC5 cells. Total fluorescence intensity of the CNA35-AF488 using the collagen probe assay (Fig. 2B) was highly correlated with increasing insulin concentration $\left(R^{2}=0.8, p=0.0066\right)$. Using the GAG assay (Fig. $\left.2 \mathrm{C}\right)$, total GAG content and insulin concentration were less correlated $\left(R^{2}=0.37, p=0.1442\right)$. Thus, a collagen probe assay has better resolution in measuring the response of ATDC5 cells to changes in stimuli.

\section{Screening for compounds enhancing ECM production by ATDC5 cells}

To find small molecules capable of enhancing cECM production by ATDC 5 cells, we screened the LOPAC1280 library (containing 1280 different compounds) using total fluorescence intensity of the collagen probe CNA35-AF488 staining as readout (Supplementary Fig. S1A). Supplementary Figure S1B shows the mean and standard deviation of the PC and NC in every plate. Total cECM content was significantly increased $(p<0.0001)$ when insulin was added $(1.5$-fold increase in total fluorescence intensity: $\mathrm{NC}=15264 \pm 1552$ vs. $\mathrm{PC}=22,718 \pm$ 1294). Since insulin is a very potent cECM inducer for ATDC5 cells, no compound was able to induce cECM secretion to a higher level than that of the IM in this screen. Thus, we decided to define hits based on their ability to enhance $\mathrm{cECM}$ production to a significantly higher level than BM, for which a hit selection threshold was chosen as the mean plus three times the standard deviation of the negative controls in the same plate. Based on this criterion, we identified in total 13 hit compounds (Supplementary Table S1), 6 of which were ordered for further testing. Selected hits were further tested using a wider range of concentrations $(2-200 \mu \mathrm{M})$ to confirm the cECM-inducing effect on ATDC5 cells. As in the HTS assay, BM and IM were used as NC and PC, respectively. Metabolic activity assay was performed to examine potential cytotoxic effects of the compounds. Of the six compounds tested, only DL-Cycloserine
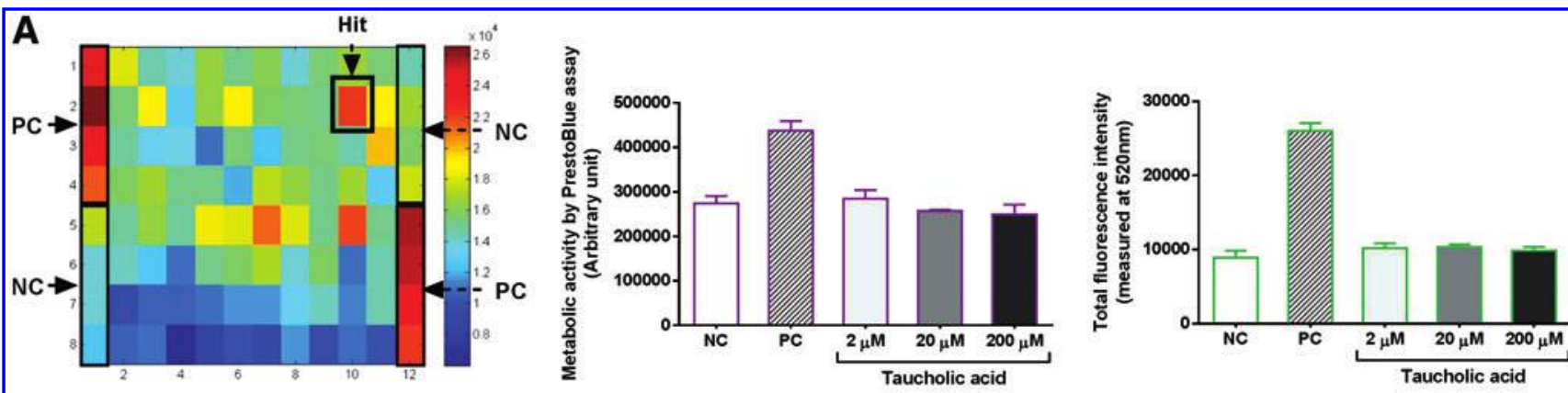

B
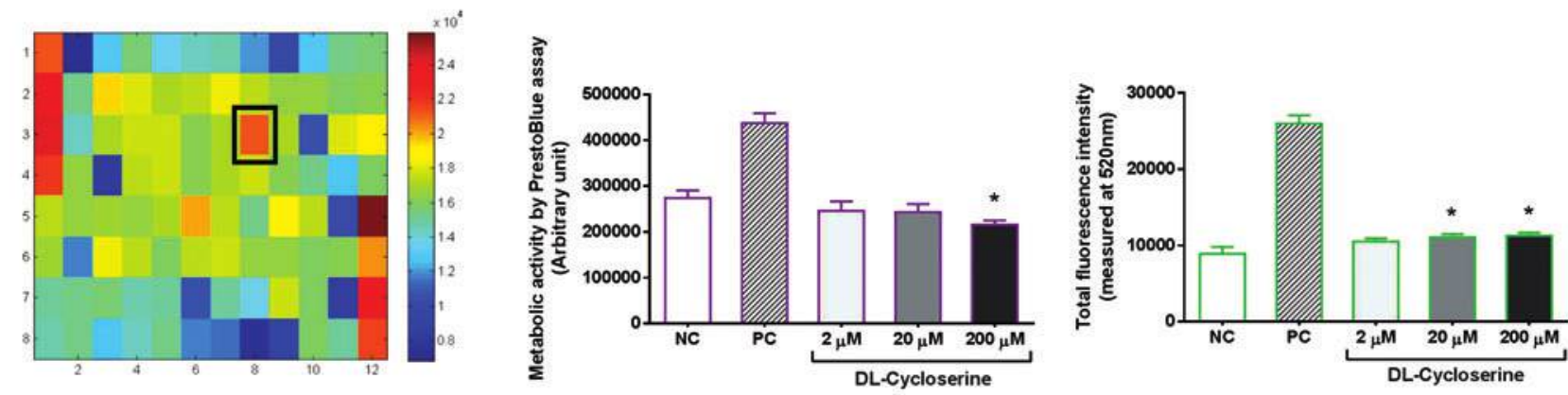

C
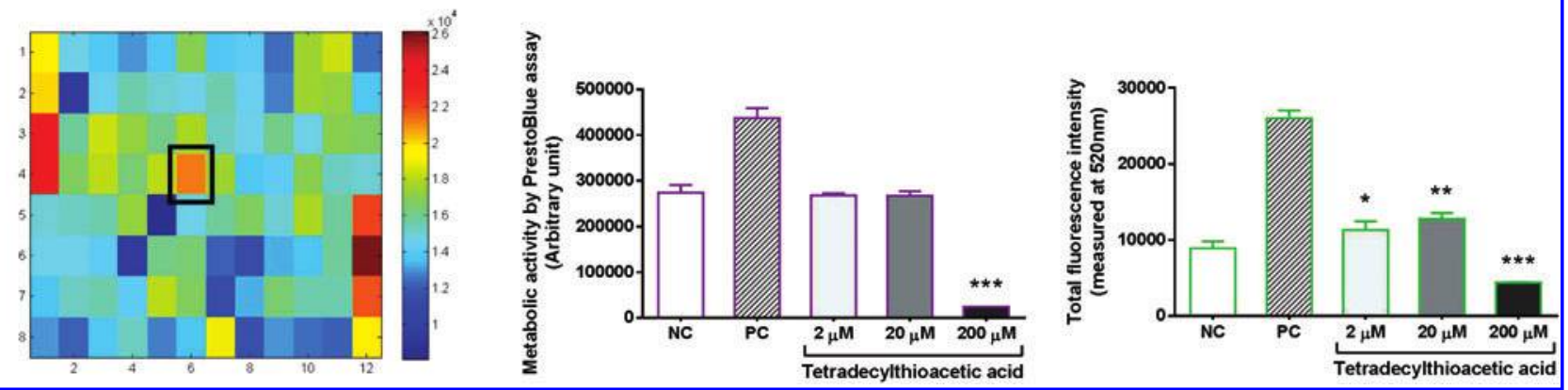

FIG. 3. Hit identification and validation. Representative figures show how hits were identified and validated for (A)-plate 1 (identify taucholic acid), (B)-plate 4 (identify DL-Cycloserine), and (C)-plate 15 (identify tetradecylthioacetic acid). Plate number is specified in the LOPAC ${ }^{1280}$ catalog. Heat maps (left) show relative total fluorescence intensity of each well on the plate compared with the PC and NC. Bar graphs (middle) show the effects of different concentrations of hit compounds on metabolic activity of ATDC5. Bar graphs (right) validate the capability of hit compounds in different contractions to enhance collagenous ECM production of ATDC5, quantified by the collagen probe assay. Error bars represent standard deviation $(n=3)$. Statistical analysis was performed using one-way ANOVA and Bonferroni's post-test. $(*)$ denotes $p<0.05$ compared with $\mathrm{NC},(* *)$ denotes $p<0.01$ compared with $\mathrm{NC}$, and $(* * *)$ denotes $p<0.001$ compared with $\mathrm{NC}$. Color images available online at www.liebertpub.com/tec 
and tetradecylthioacetic acid (TTA) were able to reproduce the cECM-inducing effect found on the HTS assay, inducing a significantly higher amount of $\mathrm{CECM}$ compared with the NC (Fig. 3B, C).

\section{Expression of ECM genes}

Next, we investigated the effect of the selected compounds on ATDC5 cells at the mRNA level. There are many different cECM proteins, but we were mostly interested in collagen type II and aggrecan since these cECM proteins form the major structural component of cartilage, especially articular cartilage. ATDC5 cells were cultured in the presence of the selected compounds for 10 days. The concentrations tested were the ones that induced the highest amount of cECM in the previous validation experiment. IM was again used as a positive control and compared with BM, it upregulated collagen type II expression 2.5 times and
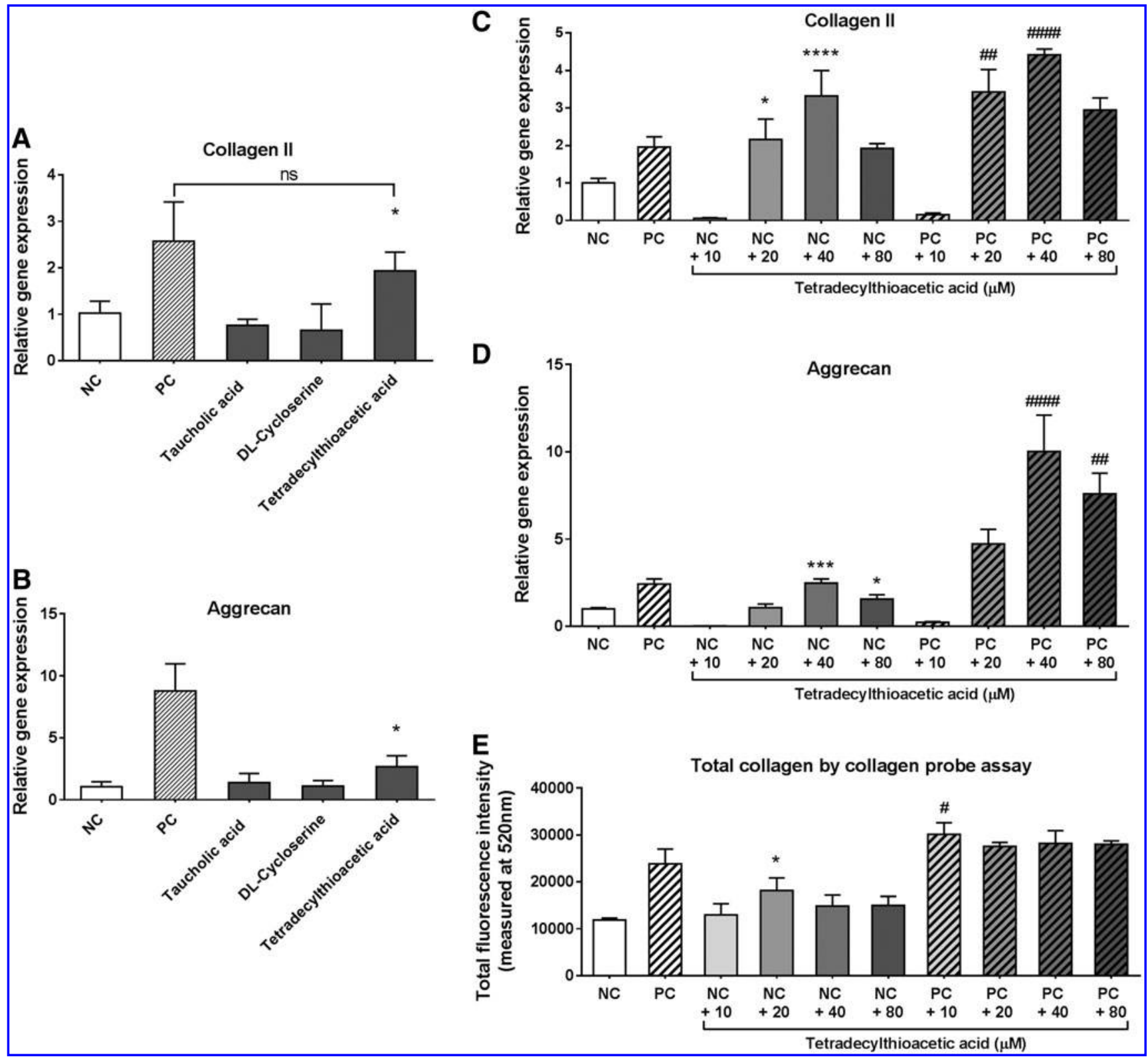

FIG. 4. Further validation of hit compounds by gene expression analysis. Collagen type II expression (A) and aggrecan expression (B) of ATDC5 treated with the hit compounds for 10 days. The concentration of the compounds was determined from the previous validation experiments. Only tetradecylthioacetic acid (TTA) showed a significant difference compared with the NC and thus was further tested in a dose-response experiment. (C-E) Dose-response effect of TTA on ATDC5. TTA was added at different concentrations to BM and IM. Collagen II expression (C), aggrecan expression (D), and total collagen quantification by collagen probe assay $(\mathbf{E})$ were measured after 10 days. Notably, when combined with IM, TTA exerted a synergistic effect on aggrecan expression of ATDC5. Error bars represent standard deviation $(n=3)$. Statistical analysis was performed using one-way ANOVA and Bonferroni's post-test. (*) denotes a significant difference compared with the BM and (\#) denotes a significant difference compared with the IM. $(*)$ or $(\#)$ denotes $p<0.05,(* *)$ or $(\# \#)$ denotes $p<0.01,(* * *)$ denotes $p<0.001$, and (****) or (\#\#\#) denotes $p<0.0001$.\#\#\#, ns, non-significant. 
aggrecan 9 times. From the tested compounds, only $20 \mu \mathrm{M}$ TTA was able to induce a significantly higher expression of collagen type II (2-fold) and aggrecan (2.6-fold) compared with the BM (Fig. 4A, B). Based on this result and the results from the previous experiment, we decided to select TTA for further testing.

To further explore the effect of TTA on ATDC5 cells, we tested TTA in a narrower range of concentrations spanning the $20 \mu \mathrm{M}$ previously tested (from $10 \mu \mathrm{M}$ to $80 \mu \mathrm{M}$ ). Furthermore, we exposed cells to both TTA and insulin to see whether they could have a synergistic or additive effect on cECM production by ATDC5 cells. Interestingly, the expression of collagen type II (Fig. 4C) and aggrecan (Fig. 4D) was significantly higher when TTA was added to IM. At the protein level (using the collagen probe assay), $10 \mu \mathrm{M}$ TTA combined with IM induced a significantly higher amount of ECM compared with IM alone (Fig. 4E).

\section{Effect of TTA in long-term culture with ATDC5}

To test the effect of TTA on ATDC5 cells in long-term culture, we cultured ATDC5 cells in BM and IM with or without $20 \mu \mathrm{M}$ TTA for 4 weeks. Figure 5 shows representative pictures of the cell layers in different conditions and different time points. Over time, the cell layers in all conditions developed cell aggregations, which grew into nodules at later time points. In the presence of $20 \mu \mathrm{M}$ TTA, this process was accelerated. Moreover, with TTA, the nodules were often bigger and denser. Gene expression analysis was performed for collagen type II, collagen type $\mathrm{X}$, aggrecan, and SOX9 (Fig. 6A-D). In the presence of $20 \mu \mathrm{M}$ TTA, expression of all these genes was upregulated compared with the conditions without TTA (except for SOX9 at week 3 and 4). The upregulation was more pronounced between weeks 2 and 4 .
A hydroxyproline assay was also performed to estimate total collagen content of the ECM derived from ATDC5 cells (Fig. 6E). Although hydroxyproline content of all conditions increased consistently over time, we did not find any statistically significant difference between the TTA conditions and other conditions mostly due to the large technical variation in the different replicates. Even the difference in the amount of hydroxyproline in IM versus BM was not evident until week 4 when a statistically significant difference was observed.

\section{Effect of TTA on other cell types}

We confirmed that TTA was capable of enhancing cECM production and inducing collagen type II and aggrecan expression in ADTC5 cells. Thus, we decided to test the effect of TTA on other cell types to see whether its effect is celltype dependent. For this purpose, we chose human primary articular chondrocytes (hPCs) and hMSCs, the two most clinically relevant cell types for cell-based therapies aimed at cartilage repair. Three different donors were tested for each cell type to correct for donor variability. Basic and chondrogenic media were used as negative and positive controls, respectively. The concentration of TTA used was $10 \mu \mathrm{M}$, determined by preliminary experiments using metabolic activity assay to exclude toxic concentrations (data not shown). Since hPCs are known to produce cECM in culture, we grew them on tissue culture plastic for 14 days, and then performed gene expression analysis for collagen type II, aggrecan, and SOX9. hMSCs were chondrogenically differentiated in pellet cultures for 30 days, followed by gene expression analysis also on the same set of genes.

For hPCs, $10 \mu \mathrm{M}$ TTA combined with chondrogenic medium increased the expression of aggrecan in all three donors (statistically significant in two out of three donors).
FIG. 5. ATDC5 cell layer in long-term culture with TTA. Representative microscopy pictures of ATDC5 cultured on TCP at weeks $1-4$ in $\mathrm{BM}, \mathrm{BM}+20 \mu \mathrm{M}$ TTA, $\mathrm{IM}$, and $\mathrm{IM}+20 \mu \mathrm{M}$ TTA. Scale bar represents $1 \mathrm{~mm}$. Color images available online at www.liebertpub.com/tec

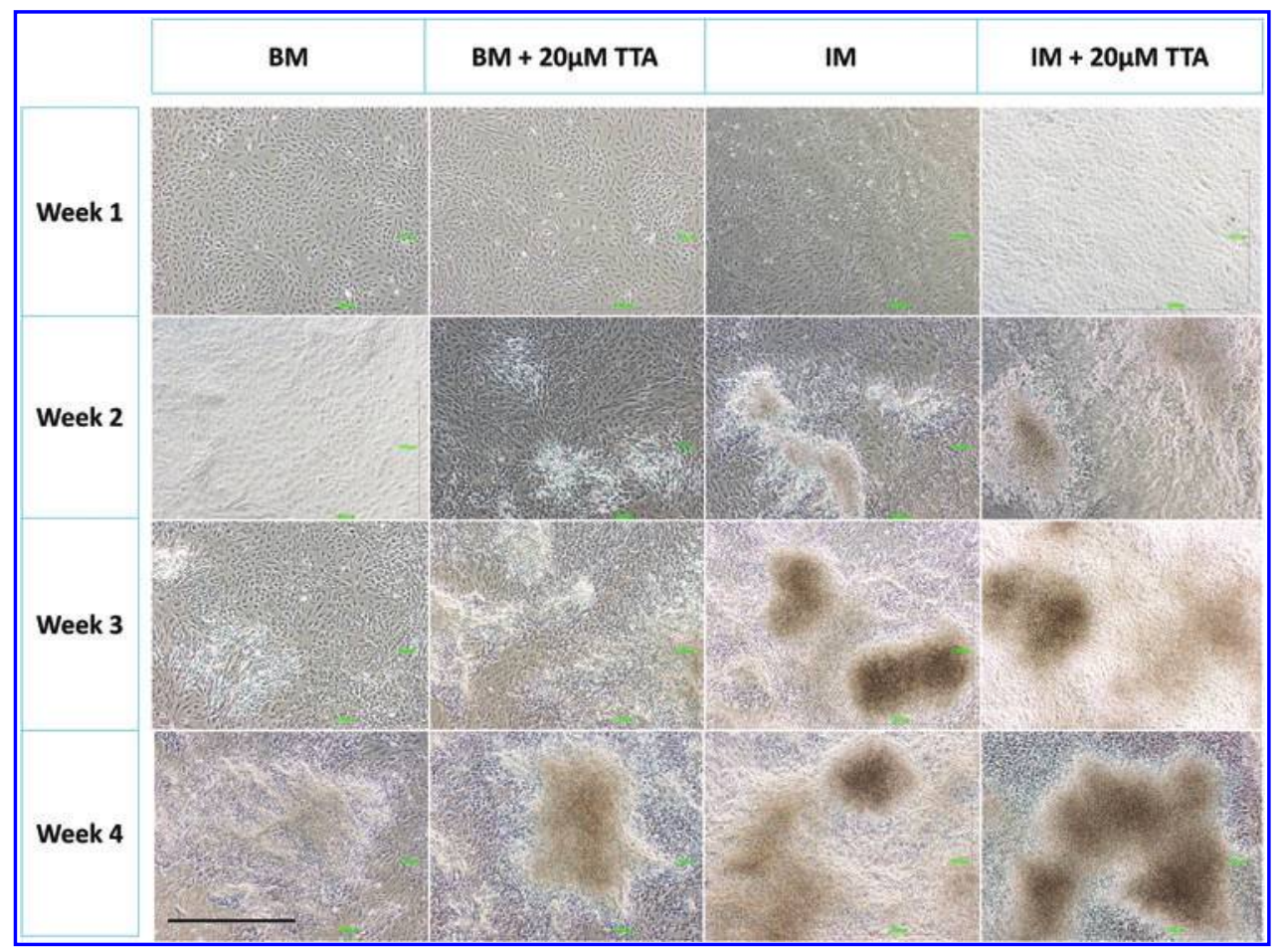




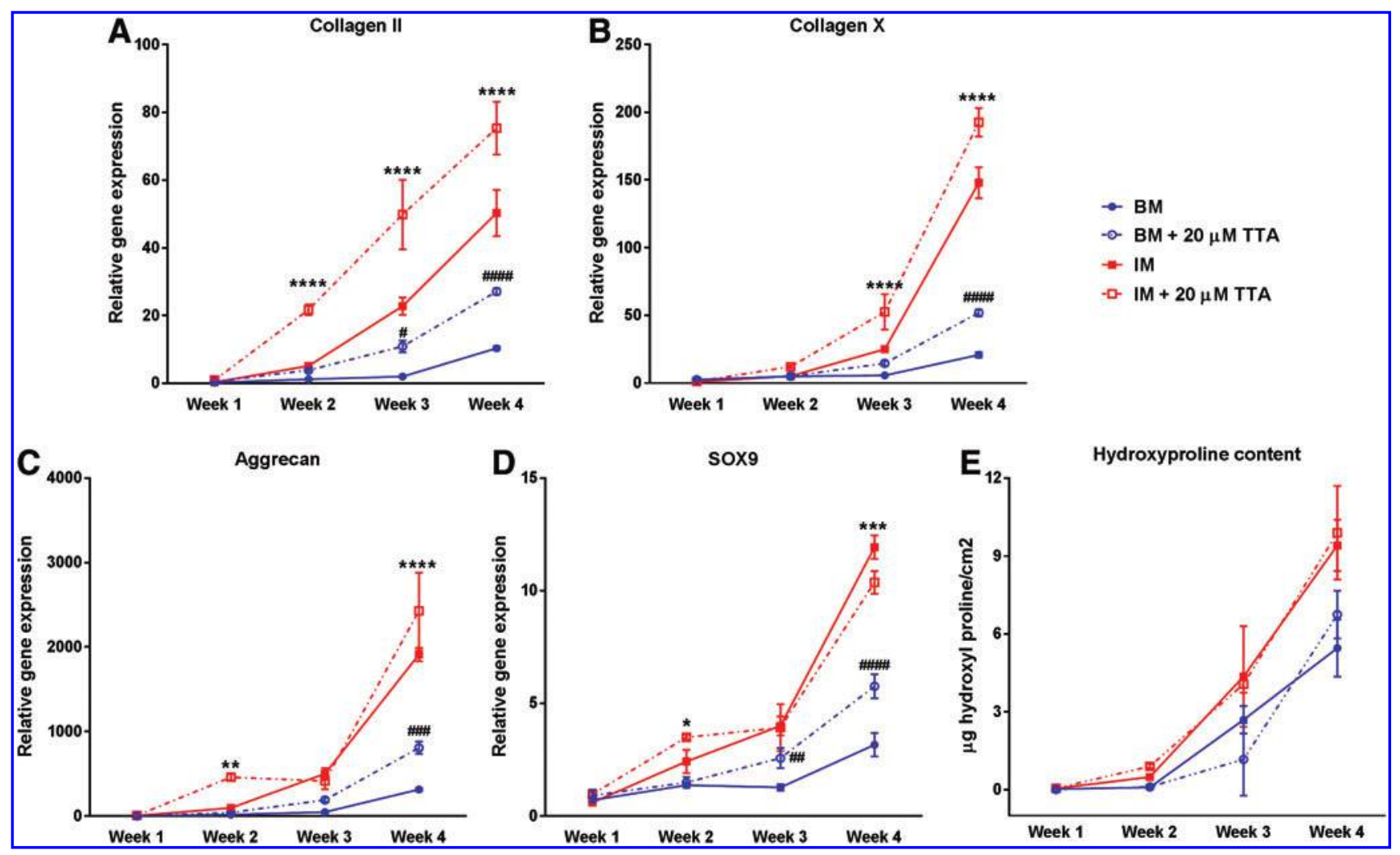

FIG. 6. The effect of TTA in long-term culture with ATDC5. Gene expression profile of collagen type II (A), collagen type X (B), aggrecan (C), and SOX9 (D), plus hydroxyproline content (E) of ATDC5 cultured on TCP in BM (solid blue line), BM supplement with $20 \mu \mathrm{M}$ TTA (broken blue line), IM (solid red line), and IM supplemented with $20 \mu \mathrm{M}$ TTA (broken red line) at weeks 1, 2, 3, and 4. Error bars represent standard deviation $(n=3)$. Statistical analysis was performed using two-way ANOVA and Bonferroni's post-test. (*) denotes a significant difference compared with the BM at the same time point and (\#) denotes a significant difference compared with the IM at the same time point. $(*)$ or $(\#)$ denotes $p<0.05,(* *)$ or $(\# \#)$ denotes $p<0.01,(* * *)$ or (\#\#\#) denotes $p<0.001$, and (****) or (\#\#\#\#) denotes $p<0.0001$. Color images available online at www.liebertpub.com/tec

However, either used alone or in combination with chondrogenic medium, TTA downregulated the expression of collagen type II and SOX9 in this cell type (Fig. 7A). Interestingly, the same trend was seen in hMSCs when $10 \mu \mathrm{M}$ TTA significantly downregulated collagen type II and SOX 9 expression in two of three donors (Fig. 7B). In the other donor (D301), collagen type II and SOX9 expression was upregulated (not significantly), and aggrecan expression increased 30-fold compared with the negative control when TTA was added. However, when combined with chondrogenic medium, TTA always reduced expression of these genes compared with the chondrogenic medium condition.

\section{Discussion}

Designing and performing an HTS requires that a number of issues are addressed. In this screen, we chose insulin as a positive control because insulin is widely used to induce ECM secretion of ATDC5 cells. + Using total fluorescent intensity of the collagen probe as a readout, even insulin was only able to induce a 1.5-fold, in total, fluorescence intensity. Still, of the six hits selected for validation from the recent screen, DL-Cycloserine and TTA could reproduce the positive response observed with insulin, although to a lesser extent. In later validation experiments, using a more sensitive technique such as qPCR with higher concentration and longer exposure time, we were able to redeem TTA as a true enhancer of cECM expression for ATDC5 cells. This could not have been done in the actual screen since all compounds were only tested at one concentration and one exposure time. Our screen was designed to be exploratory because a universal mechanism for inducing cECM protein production is not known. For this purpose, we chose a nonspecific readout: total amount of collagen estimated by total fluorescence intensity from a collagen probe staining, which is not specific for any type of collagen. The most advantageous point of the collagen probe staining is its ease of use and thus is ideal for an HTS. The three step protocol, adding the dye, washing, and quantifying, can be performed in any laboratory manually or automatically. Its main problem is the instability of the fluorophore; even though Alexa Fluor is very good, photo bleaching will always occur. We addressed this problem by performing the staining and quantification in batches and always included both the positive and negative controls on every plate. Since the collagen probe recognizes the amino acid sequence of collagens, ${ }^{16,21}$ it will also bind to them in solution. However, the complex remains in solution and will be removed in the washing step. The collagen probe can also bind to collagen gel (with chopped fiber) and gelatin (denatured collagen), thus it is not suitable to use in a gel system.

Other methods that can be used to quantify collagen include hydroxyproline assay and Picro Sirius red assay. 


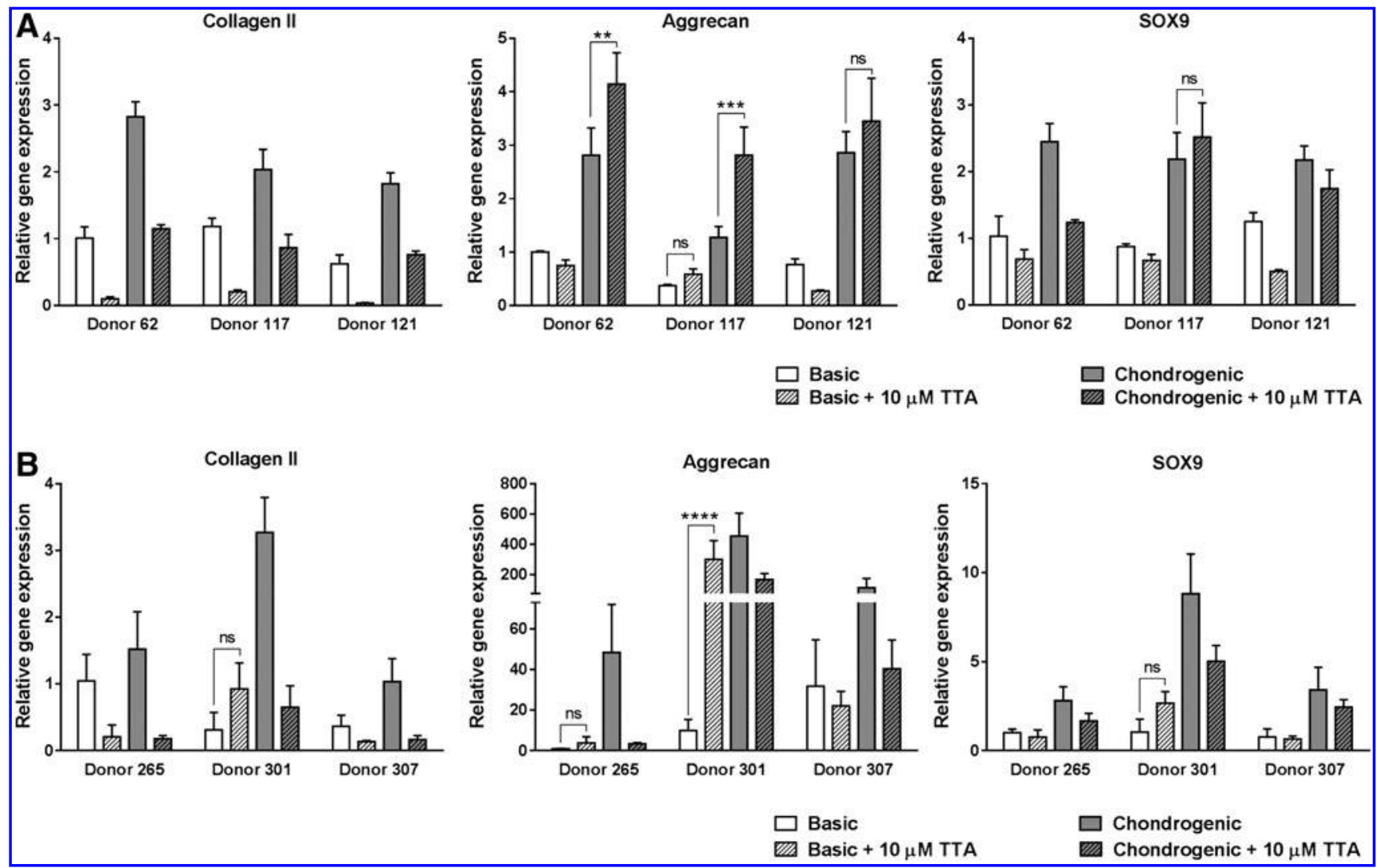

FIG. 7. Effect of TTA on human primary chondrocytes and human mesenchymal stromal cells. (A) hPCs (three donors) were cultured on TCP in chondrocyte basic medium (CBM), CBM $+10 \mu \mathrm{M}$ TTA, chondrogenic differentiation medium $(\mathrm{CDM})$, and CDM $+10 \mu \mathrm{M}$ TTA. Gene expression analysis on collagen type II, aggrecan, and SOX9 was performed after 14 days in culture. (B) hMSCs (three donors) were cultured in pellets of $2.5 \times 10^{5}$ cells in hMSC basic medium (MBM), $\mathrm{MBM}+10 \mu \mathrm{M}$ TTA, hMSC chondrogenic differentiation medium (MCM), and MCM $10 \mu \mathrm{M}$ TTA. Gene expression analysis on collagen type II, aggrecan, and $S O X 9$ was performed after 30 days in culture. Error bars represent standard deviation ( $n=3$ for each donor). Statistical analysis was performed using one-way ANOVA and Bonferroni's post-test for each donor. $(* *)$ denotes $p<0.01$, (***) denotes $p<0.001$, and $(* * * *)$ denotes $p<0.0001$, ns, nonsignificant.

While the hydroxyproline is by far the most common method for quantifying tissue fibrosis and collagen deposition, ${ }^{22}$ its laborious protocol hinders its high-throughput capability. The hydrolysis step, which is necessary for the release of amino acid hydroxyproline from the protein collagen, requires high temperature and high pressure and thus is not suitable for normal plastic well plates. With some modifications, the Picro Sirius red staining can be used in an HTS very similar to our collagen probe assay. ${ }^{23}$ When bound to collagen, the Sirius red-collagen complex will precipitate, making it suitable for quantifying even soluble collagens.

While we confirmed that TTA is capable of enhancing cECM production by ATDC5 cells, we did not attempt to study the mechanism behind it. To date, TTA is a well-known synthetic fatty acid and is the focus of many studies for its effect on fat metabolism-related disorders, ${ }^{24}$ inflammation, ${ }^{25}$ and even cardiovascular function. ${ }^{26,27}$ A lot of evidence points at the involvement of TTA in the peroxisome proliferator-activated receptor (PPAR)-mediated signaling. ${ }^{28-30}$ According to Forman et al., ${ }^{30}$ TTA belongs to a group of dual-function PPAR $\alpha$ activators, which also includes tetradecylthioproprionic acid (TTP), tetradecylglycidic acid (TDGA), octylthioproprionic acid (OTP), nonylthioacetic acid (NTA), LY 171883, and 2-bromopalmitate (2Br-C16).
All these compounds activate PPAR $\alpha$ directly as ligands; plus, as metabolic inhibitors of the mitochondrial $\beta$-oxidation pathway, they cause accumulation of endogenous fatty acids, which are also PPAR $\alpha$ ligands.

Some in vitro researches have shown the antiproliferative effect of TTA on certain cell types, ${ }^{31-33}$ which was not confirmed by our data. Exposure of ATDC5 cells, hMSCs, and hPCs to TTA did not have a noticeable negative effect on proliferation unless very high concentrations of TTA $(200 \mu \mathrm{M}$ for ATDC5, $40 \mu \mathrm{M}$ for hMSCs and hPCs) were used, which led to a reduction in metabolic activity, followed by cell death (Fig. 3C, middle graph).

Since ATDC5 is a chondrogenic cell line, we wanted to see the same cECM-enhancing effect of TTA on hPCs, but unexpectedly, in our experimental conditions, TTA consistently inhibited the expression of collagen II in hPCs. This was also observed for hMSCs undergoing chondrogenic differentiation. We have yet to explain this phenomenon, but the fact that ATDC5 cells can produce abundant cECM over time even in BM, suggests that the mechanism of action of TTA on ATDC5 cells is different from that on hMSCs and hPCs. Westergaard et al. showed that TTA activated all human PPAR subtypes in the ranking order PPAR $\delta>>$ PPAR $\alpha$ $>$ PPAR $\gamma$, whereas in the experiment using mouse PPARs, 
TTA most efficaciously activated PPAR $\alpha .^{33}$ Although this could not explain the contradictory effect that we have seen in our experiments, it showed that the mechanism of action of TTA may be different between species.

With the ECM-enhancing effect of TTA on ATDC5 cells confirmed, especially when used in combination with insulin, we obtained a new method to grow and upscale our ATDC5 cartilage model in vitro. With our initial plan of studying the endochondral ossification process using live or devitalized hypertrophic cartilage, we can now use TTA in combination with insulin to enhance the amount of tissue mass produced by ATDC5 for in vivo implantation without the need of scaffold material. While increasing collagen quantity is advantageous for our purpose, collagen structure and organization can greatly affect property and functionality of the ECM. In previous publications, ${ }^{16,17}$ we have shown the capability of the collagen probe in high-resolution imaging. Collagen organization can be visualized with the collagen probe even better than with second harmonic generation, which is currently the most effective method for viewing 3D collagen organization in tissues. However, this resolution is not high enough to visualize collagen fibril periodicity. In this article, we have yet to look at collagen structure and organization. In the near future, we will focus on using micropatterned surfaces to modify the collagen fibril organization of the ATDC5 cell layer.

In conclusion, we have developed an HTS assay using the collagen probe CNA35-AF488 to screen for compounds capable of enhancing CECM production by the cells, and as an example, we discovered TTA as a potent cECM enhancer of ATDC5. With the method we present, any laboratory can customize the screen by choosing a different target cell type (e.g., primary chondrocytes, hMSCs) and a different compound library; the result will be different compounds that respond well to their cell type in terms of collagen production.

\section{Disclosure Statement}

No competing financial interests exist.

\section{References}

1. Guilak, F., et al. Control of stem cell fate by physical interactions with the extracellular matrix. Cell Stem Cell 5, 17, 2009.

2. Watt, F.M., and Hogan, B.L. Out of Eden: stem cells and their niches. Science 287, 1427, 2000.

3. Unadkat, H.V., et al. An algorithm-based topographical biomaterials library to instruct cell fate. Proc Natl Acad Sci U S A 108, 16565, 2011.

4. von der Mark, K., et al. Nanoscale engineering of biomimetic surfaces: cues from the extracellular matrix. Cell Tissue Res 339, 131, 2010.

5. Moroni, L., de Wijn, J.R., and van Blitterswijk, C.A. Integrating novel technologies to fabricate smart scaffolds. J Biomater Sci Polym Ed 19, 543, 2008.

6. Jukes, J.M., et al. Endochondral bone tissue engineering using embryonic stem cells. Proc Natl Acad Sci U S A 105, 6840, 2008.

7. Bridges, J.B., and Pritchard, J.J. Bone and cartilage induction in the rabbit. J Anat 92, 28, 1958.
8. Urist, M.R., and Mc, L.F. Osteogenetic potency and newbone formation by induction in transplants to the anterior chamber of the eye. J Bone Joint Surg Am 34-A, 443, 1952.

9. Urist, M.R., Wallace, T.H., and Adams, T. The function of fibrocartilaginous fracture callus. Observations on Transplants Labelled with Tritiated Thymidine. J Bone Joint Surg Br 47, 304, 1965.

10. Urist, M.R., and Adams, T. Cartilage or bone induction by articular cartilage. Observations with radioisotope labelling techniques. J Bone Joint Surg Br 50, 198, 1968.

11. Muratov, R., et al. New approach to reduce allograft tissue immunogenicity. Experimental data. Interact Cardiovasc Thorac Surg 10, 408, 2010.

12. DeLustro, F., et al. Immune responses to allogeneic and xenogeneic implants of collagen and collagen derivatives. Clin Orthop Relat Res 260, 263, 1990.

13. Alves, H., et al. High-throughput assay for the identification of compounds regulating osteogenic differentiation of human mesenchymal stromal cells. PLoS ONE 6, e26678, 2011.

14. Doorn, J., et al. A small molecule approach to engineering vascularized tissue. Biomaterials 34, 3053, 2013.

15. Shukunami, C., et al. Cellular hypertrophy and calcification of embryonal carcinoma-derived chondrogenic cell line ATDC5 in vitro. J Bone Miner Res 12, 1174, 1997.

16. Krahn, K.N., et al. Fluorescently labeled collagen binding proteins allow specific visualization of collagen in tissues and live cell culture. Anal Biochem 350, 177, 2006.

17. Boerboom, R.A., et al. High resolution imaging of collagen organisation and synthesis using a versatile collagen specific probe. J Struct Biol 159, 392, 2007.

18. Saito, T., et al. Transcriptional regulation of endochondral ossification by HIF-2alpha during skeletal growth and osteoarthritis development. Nat Med 16, 678, 2010.

19. Alves, H., et al. Effect of antioxidant supplementation on the total yield, oxidative stress levels, and multipotency of bone marrow-derived human mesenchymal stromal cells. Tissue Eng Part A 19, 928, 2013.

20. Hendriks, J.A., et al. Primary chondrocytes enhance cartilage tissue formation upon co-culture with a range of cell types. Soft Matter 6, 5080, 2010.

21. Patti, J.M., Boles, J.O., and Hook, M. Identification and biochemical characterization of the ligand binding domain of the collagen adhesin from Staphylococcus aureus. Biochemistry 32, 11428, 1993.

22. Woessner, J.F., Jr., The determination of hydroxyproline in tissue and protein samples containing small proportions of this imino acid. Arch Biochem Biophys 93, 440, 1961.

23. Kliment, C.R., et al. A novel method for accurate collagen and biochemical assessment of pulmonary tissue utilizing one animal. Int J Clin Exp Pathol 4, 349, 2011.

24. Berge, R.K., et al. Metabolic effects of thia fatty acids. Curr Opin Lipidol 13, 295, 2002.

25. Bjorndal, B., et al. Tetradecylthioacetic acid attenuates inflammation and has antioxidative potential during experimental colitis in rats. Dig Dis Sci 58, 97, 2013.

26. Pettersen, R.J., et al. Effects of local delivery of tetradecylthioacetic acid within the injured coronary vessel wall. Scand Cardiovasc J 46, 366, 2012.

27. Oie, E., et al. Tetradecylthioacetic acid increases fat metabolism and improves cardiac function in experimental heart failure. Lipids 48, 139, 2013.

28. Wrzesinski, K., et al. Proteomics identifies molecular networks affected by tetradecylthioacetic acid and fish oil supplemented diets. J Proteomics 84, 61, 2013. 
29. Bocos, C., et al. Fatty acid activation of peroxisome proliferator-activated receptor (PPAR). J Steroid Biochem Mol Biol 53, 467, 1995.

30. Forman, B.M., Chen, J., and Evans, R.M. Hypolipidemic drugs, polyunsaturated fatty acids, and eicosanoids are ligands for peroxisome proliferator-activated receptors alpha and delta. Proc Natl Acad Sci U S A 94, 4312, 1997.

31. Lundemo, A.G., et al. Tetradecylthioacetic acid inhibits proliferation of human SW620 colon cancer cells-gene expression profiling implies endoplasmic reticulum stress. Lipids Health Dis 10, 190, 2011.

32. Tronstad, K.J., et al. Antiproliferative effects of a non-betaoxidizable fatty acid, tetradecylthioacetic acid, in native human acute myelogenous leukemia blast cultures. Leukemia 16, 2292, 2002.

33. Westergaard, M., et al. Modulation of keratinocyte gene expression and differentiation by PPAR-selective ligands and tetradecylthioacetic acid. J Invest Dermatol 116, 702, 2001.
Address correspondence to: Bach q. Le, BSc Department of Tissue Regeneration MIRA Institute for Biomedical Technology and Technical Medicine University of Twente Postbus 217

Enschede 7500 AE

The Netherlands

E-mail: b.q.le@tnw.utwente.nl

Received: February 3, 2014

Accepted: December 16, 2014

Online Publication Date: April 6, 2015 


\section{This article has been cited by:}

1. Farhad Soheilmoghaddam, Madeleine Rumble, Justin Cooper-White. 2021. High-Throughput Routes to Biomaterials Discovery. Chemical Reviews 43. . [Crossref]

2. Steven Vermeulen, Jan de Boer. 2021. Screening as a strategy to drive regenerative medicine research. Methods $190,80-95$. [Crossref]

3. Nicola C. Foster, Nicole M. Hall, Alicia J. El Haj. Two-Dimensional and Three-Dimensional Cartilage Model Platforms for Drug Evaluation and High-Throughput Screening Assays. Tissue Engineering Part B: Reviews, ahead of print. [Abstract] [Full Text] [PDF] [PDF Plus]

4. Dr. Nicola Carrinne Foster, Miss Nicole M Hall, Prof. Alicia J El Haj. 2D and 3D cartilage model platforms for drug evaluation and high-throughput screening assays. Tissue Engineering Part B: Reviews 0:ja. . [Abstract] [PDF] [PDF Plus]

5. James E. Dennis, Taylor Splawn, Thomas J. Kean. 2020. High-Throughput, Temporal and Dose Dependent, Effect of Vitamins and Minerals on Chondrogenesis. Frontiers in Cell and Developmental Biology 8. . [Crossref]

6. Madeline Y. Wong, Ngoc Duc Doan, Andrew S. DiChiara, Louis J. Papa, Jaime H. Cheah, Christian K. Soule, Nicki Watson, John D. Hulleman, Matthew D. Shoulders. 2018. A High-Throughput Assay for Collagen Secretion Suggests an Unanticipated Role for Hsp90 in Collagen Production. Biochemistry 57:19, 2814-2827. [Crossref]

7. Ghebes Corina Adriana, Groen Nathalie, Cheuk Yau Chuk, Fu Sai Chuen, Fernandes Hugo Machado, Saris Daniel B.F.. 2018. Muscle-Secreted Factors Improve Anterior Cruciate Ligament Graft Healing: An In Vitro and In Vivo Analysis. Tissue Engineering Part A 24:3-4, 322-334. [Abstract] [Full Text] [PDF] [PDF Plus] [Supplementary Material]

8. Jose Diaz-Romero, Sibylle Kürsener, Sandro Kohl, Dobrila Nesic. 2017. S100B + A1 CELISA: A Novel Potency Assay and Screening Tool for Redifferentiation Stimuli of Human Articular Chondrocytes. Journal of Cellular Physiology 232:6, 1559-1570. [Crossref]

9. Le Bach Q., Vasilevich Aliaksei, Vermeulen Steven, Hulshof Frits, Stamatialis Dimitrios F., van Blitterswijk Clemens A., de Boer Jan. 2017. Micro-Topographies Promote Late Chondrogenic Differentiation Markers in the ATDC5 Cell Line. Tissue Engineering Part A 23:9-10, 458-469. [Abstract] [Full Text] [PDF] [PDF Plus] [Supplementary Material] 